\title{
N18 in median somatosensory evoked potentials: a new indicator of medullary function useful for the diagnosis of brain death
}

\author{
Masahiro Sonoo, Yasunobu Tsai-Shozawa, Makoto Aoki, Toshio Nakatani, \\ Yuuki Hatanaka, Atsuko Mochizuki, Mikio Sawada, Kunio Kobayashi, Teruo Shimizu
}

Department of Neurology M Sonoo

Y Tsai-Shozawa

Y Hatanaka

A Mochizuki

M Sawada

T Shimizu

Department of Neurosurgery

M Aoki

Trauma and Critical Care Center, Teikyo University School of Medicine, Tokyo, Japan T Nakatani

K Kobayashi

Correspondence to: Dr Masahiro Sonoo, Department of Neurology, Teikyo University School of Medicine, Kaga 2-11-1, Itabashi-ku, Tokyo 173, Japan. Telephone 00813 39641211 ext 1911; fax 008133964 6397; email sonoom@med.teikyo-u.ac.jp

Received 8 January 1999 and in revised form

7 April 1999

Accepted 13 April 1999

\begin{abstract}
Objectives-To record $\mathrm{N} 18$ in median somatosensory evoked potentials (SEPs) for deeply comatose or brain dead patients and to demonstrate the usefulness of $\mathrm{N} 18$ for the diagnosis of brain death in comparison with auditory brain stem responses (ABRs) and P13/14 in median SEPs, which have been conventionally used as complementary tests for the diagnosis of brain death.

Methods-Subjects were 19 deeply comatose or brain dead patients. Thirteen recordings were performed in deeply comatose but not brain dead conditions, and 12 recordings were performed in brain death. N18 was evaluated in the CPi-C2S lead (or other scalp-C2S leads) to obtain a flat baseline.

Results-N18 was preserved in 12 of 13 non-brain dead comatose recordings whereas it was completely lost for all of the 12 brain death recordings. P13/14 in median SEPs was preserved for all the comatose recordings, whereas apparent P13/14-like potentials, usually of low amplitude, were seen in nine of 12 brain death recordings-that is, frequent false positives. The ABRs already showed features which were characteristic for brain death (loss of components other than wave 1 or small wave 2) for four comatose recordings, in three of which N18 was preserved. The last result not only corresponds with the fact that ABRs can evaluate pontine and midbrain functions and not medullary function, but further supports the medullary origin of N18. In the four patients followed up for the course of progression from coma to brain death, N18s preserved in normal size during the comatose state were completely lost after brain death was established.

Conclusions-The N18 potential is generated by the cuneate nucleus in the medulla oblongata in the preceding studies. N18 is suggested to be a promising tool for the diagnosis of brain death because there were no false positives and rare false negatives in the present series for detecting the remaining brain stem function. (F Neurol Neurosurg Psychiatry 1999;67:374-378)
\end{abstract}

Keywords: somatosensory evoked potentials; N18; brain death; cuneate nucleus
Evoked potentials have been used as noninvasive complementary tests for the diagnosis of brain death. Among them, auditory brain stem responses (ABRs) have been the most popular. ${ }^{1-5}$ However, one drawback of ABRs is that they are generated within the pons and midbrain and do not reflect the function of the medulla oblongata, which is critical for the maintenance of vital functions such as respiration and circulation. Application of somatosensory evoked potentials (SEPs) to the diagnosis of brain death has also been investigated, mainly focusing on the $\mathrm{P} 13 / 14$ potential in median SEPs of possible brain stem origin, with limited success. ${ }^{2}{ }^{3}$ 6-10

N18 in median nerve SEPs is a broad negativity widely distributed over the scalp after positive far field potentials. ${ }^{11}$ There has been much controversy regarding its origin, but we recently presented an opinion that N18 is principally generated at the cuneate nucleus located in the caudal medulla oblongata. ${ }^{12-15}$ This hypothesis prompted us to evaluate the usefulness of N18 for the diagnosis of brain death. In this report, we present the first results of our study recording N18s in deeply comatose or brain dead patients, focusing on the comparison with ABRs and P13/14.

\section{Patients and methods}

Subjects were 19 deeply comatose (Glasgow coma scale score $\leqslant 6)$ or brain dead patients (12 men and seven women, age 20-79). Their clinical backgrounds were six with supratentorial haemorrhage, two with brain stem haemorrhage, four with subarachnoid haemorrhage, one with superior sagittal sinus thrombosis, two with brain injury, and four with brain hypoxia. Brain death was diagnosed according to the Japanese guideline, ${ }^{16}$ although the apnoea test was not usually done because the performance of a potentially invasive apnoea test for patients not intended for organ transplantation is not ethically acceptable according to the ethics committee of our university (Organ transplantation from brain dead patients was recently been allowed by law but has rarely been performed in Japan.) In this sense, there remains the theoretical possibility that the patients classified as brain dead according to our criteria excluding the apnoea test may manifest preserved respiratory function. However, we can safely assume that such cases must be very rare, because previous large series indicate that there was virtually no case in which the apnoea test disclosed masked res- 


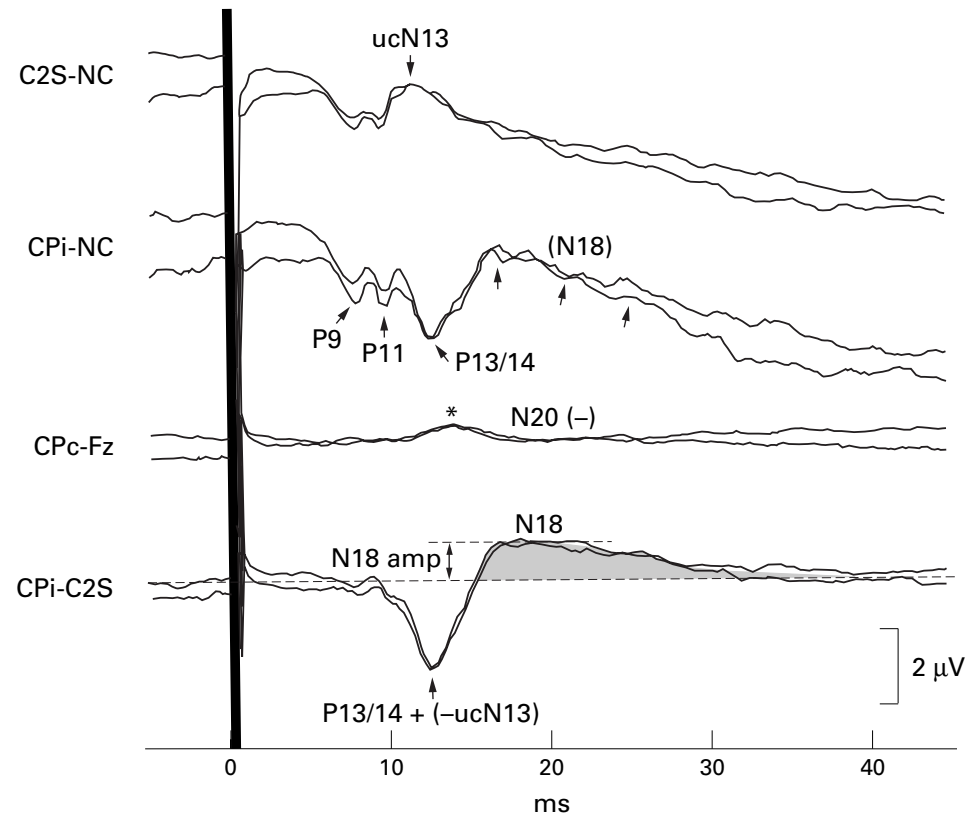

Figure 1 Example of SEPs in the comatose but not brain dead condition. In the CPi-NC lead, three far field potentials, P9, P11, and P13/14, were seen and N18 followed P13/14, although it was difficult to convincingly judge the presence of N18 or to evaluate its amplitude because of the incline of baseline due to a slow ECG artifact. By contrast, the $\mathrm{CPi}-\mathrm{C} 2 \mathrm{~S}$ lead gave a sufficiently flat baseline which enables us to correctly identify the long lasting N18 potential. N18 amplitude was measured in this lead from the baseline, which was determined in reference to the baseline before P9 and prestimulus baseline. N18 onset was continuous from the ascending limb of the preceding positivity, which is a complex potential where P13/14 in the Cpi electrode and upper cervical N13 (ucN13) in the C2S electrode 20 (reversed) are summated. N20 or other cortical components were not identified in the $\mathrm{CPc}-\mathrm{Fz}$ lead. The small negativity in this lead (asterisk) roughly corresponding to the latency of P13/14 is a potential generated because the overall P13/14 potential is deeper in the frontal electrodes, and we named this potential "broad N13" in our previous report. ${ }^{21}$

piratory function in patients strictly fulfilling other criteria of brain death. ${ }^{17}{ }^{18}$ Moreover, the point of establishment of brain death was usually clinically definable in the present series by the ceasing of spontaneous respiration accompanied by a sudden fall in blood pressure without any circulatory cause. Recovery of spontaneous respiration was never found in such patients.

Twenty five recordings were performed for these patients, of which 13 were in deeply comatose but not brain dead conditions (Brain death was excluded by preserved spontaneous respiration in 10 recordings and by constricted and reactive pupil, decerebrate response, or involuntary facial myoclonus in three other recordings.) and 12 in brain death thus clinically defined. We repeated recordings two

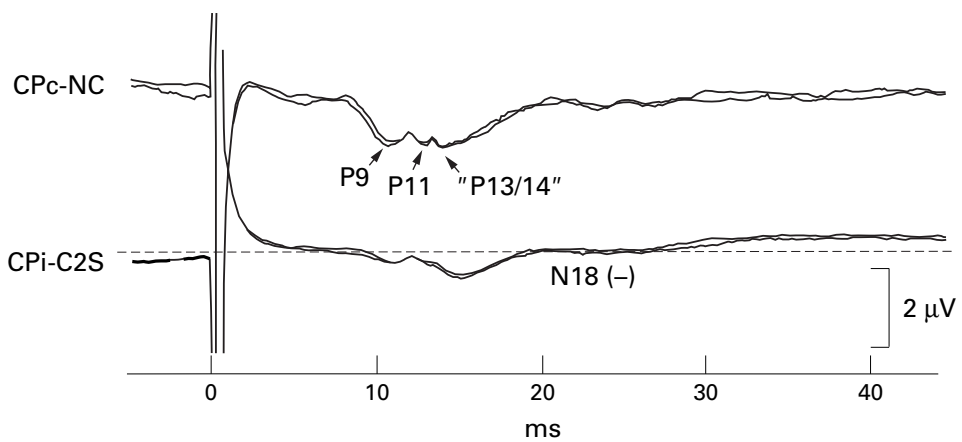

Figure 2 Preserved P13/14 in a brain dead patient. Apparent P13/14 potential was seen in the CPc-NC lead after P9 and P11 far field potentials. N18 was judged to be lost in the $C P i-C 2 S$ lead because the wave returned to the baseline after the preceding positivity. The slight negative deflection after 27 ms was not interpreted as N18. to four times during the course of progression from coma to brain death in four patients. Informed consent was obtained from the families of the patients.

The SEPs were recorded after median nerve stimulation at the wrist at a rate of $5 \mathrm{~Hz}$. The side of stimulation was selected so that the less affected side of the brain would be stimulated when there was laterality of the lesion. Recording electrodes were placed on several points including C2S (second cervical vertebrae), $\mathrm{CPc}$, and $\mathrm{CPi}$ (centroparietal electrodes contralateral and ipsilateral to the stimulated limb, respectively, as defined by the guideline of the American Electroencephalographic Society ${ }^{19}$ ), $\mathrm{Fz}$, and NC (non-cephalic reference on the contralateral shoulder). Evoked potentials were amplified and filtered between 5 and $1500 \mathrm{~Hz}$ ( $-3 \mathrm{~dB})$. Totals of $1000-4000$ (usually 2000) responses were averaged and two averages were superimposed.

$\mathrm{N} 18$ was evaluated by the CPi-C2S or other scalp-C2S leads, the second being used when cortical components were lost. These derivations with $\mathrm{C} 2 \mathrm{~S}$ reference are crucial to the evaluation of $\mathrm{N} 18$ because they give sufficiently flat baselines which enable us to correctly identify the long lasting N18 potentials. ${ }^{13}{ }^{14}$ The baseline of scalp-NC leads, which have been conventionally used to record N18, often shows a constant incline due to slow ECG artifacts (fig 1). P13/14 was evaluated by the CPc-NC or other scalp-NC leads.

$\mathrm{N} 18$ was judged to be preserved only when there was a definite increase over the baseline continuous from the ascending limb of the preceding positivity, which is a complex potential contributed by scalp P13/14 and upper cervical $\mathrm{N} 13 .{ }^{20} \mathrm{~N} 18$ thus had a rather steep onset and reached its maximal amplitude within several milliseconds of the first upward crossing over the baseline, and then slowly returned to the baseline around 20 milliseconds. The amplitude of N18 was measured from the baseline, usually within several milliseconds of its onset as described above (fig 1). The duration of N18 was also measured although the end of N18 was sometimes difficult to define accurately. In some brain death recordings, the wave returned to baseline after the preceding positivity and then showed a slight increase over the baseline after several milliseconds (fig 2). Such a negativity was not interpreted as N18 in the present study and N18 was judged to be lost in such cases. Although we cannot completely ignore the possibility that such a delayed negativity is a delayed and depressed N18, it was so different from the typical configuration of N18 and was difficult to distinguish from a residual minimal incline of the baseline.

The ABRs were recorded after a $105 \mathrm{~dB}$ NHL click sound given to both ears at a rate of $13 \mathrm{~Hz}$. Fz-A1 and Fz-A2 leads were recorded ( $F z$ was substituted for $\mathrm{Cz}$ to spare the number of electrodes). Totals of 2000-4000 responses were averaged and two averages were superimposed. 
Summary of the evoked potential findings

\begin{tabular}{lcl}
\hline & $\begin{array}{c}\text { Comatose but not } \\
\text { brain dead }(n=13)\end{array}$ & $\begin{array}{l}\text { Brain death } \\
(n=12)\end{array}$ \\
\hline SEP: & & \\
N18 & $12 / 13$ & $0 / 12$ \\
P13/14 & $13 / 13$ & $9 / 12$ \\
N20 & $3 / 13$ & $0 / 12$ \\
ABR: & & \\
$\quad$ No response & $1 / 13$ & $9 / 12$ \\
I only & $0 / 13$ & $1 / 12$ \\
$\quad$ I and small II only & $3 / 13$ & $2 / 12$ \\
$\quad$ More & $9 / 13$ & $0 / 12$ \\
\hline
\end{tabular}

\section{Results}

The results are summarised in the table. N18 was definitely preserved for all but one of the comatose recordings. The only comatose recording with absent $\mathrm{N} 18$ was found in a patient with pontine haemorrhage, in whom involuntary facial myoclonus excluded brain death but spontaneous respiration and all the brain stem reflexes were already lost, suggesting very limited survival of brain stem function. N18 was lost for all the brain death recordings. The amplitude of N18 was 0.94 (SD 0.38) $\mu \mathrm{V}$ (range $0.43-1.67 \mu \mathrm{V}$ ) and its duration was 25.8 (SD 3.0) $\mathrm{ms}$ (range 21-31 ms) for 12 comatose recordings with preserved N18. N18 of the above size was always definitely identifiable because no negativity exceeding $0.1 \mu \mathrm{V}$ over the baseline was identified directly after the preceding positivity for recordings in which N18 was judged to be lost.

P13/14 was preserved for all the comatose recordings, whereas apparent P13/14-like potential, usually of low amplitude, was definitely

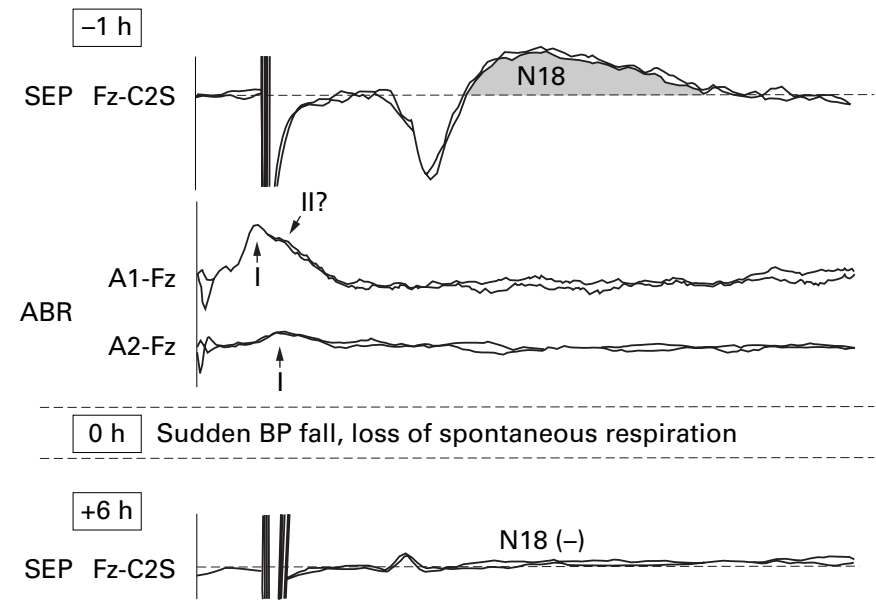

$\mathrm{ABR}$

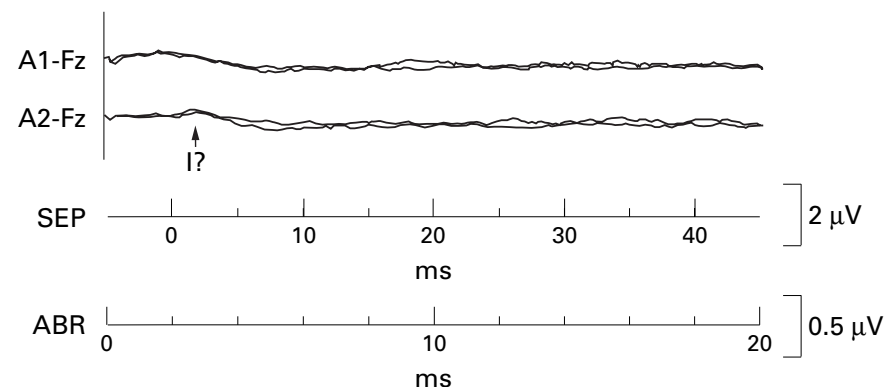

Figure 3 N18s and ABRs before and after the establishment of brain death. N18s were preserved in normal size in coma before brain death but were lost in brain death. For ABRs, bilateral wave Is and a questionable left wave II were seen in coma, whereas only a questionable right wave I was identified in brain death. found for nine out of 12 brain death recordings (fig 2). N20 was already lost for most of the comatose recordings.

The ABRs in brain death recordings were either lost (nine of 12) or registered no more than wave I and small wave II, consistent with previous reports of features of ABRs in brain death. ${ }^{25}$ However, similar ABR patterns were also found in four of 13 comatose recordings. N18 was preserved in three of four such recordings (the exception was the recording from the patient with pontine haemorrhage), from patients in whom brain death was excluded by preserved spontaneous respiration and cough reflex (two recordings) or by decerebrate response (one recording).

For all of the four patients in whom we followed the course of progression from coma to brain death, N18s preserved in normal size during the comatose state were lost after brain death was established. P13/14 was also lost after brain death was established in two of the four patients, including the example presented (fig 3). In another two patients, P13/14 greatly reduced its amplitude but was preserved in low amplitude even after brain death was established. A typical example is shown in fig 3. The patient was a 53 year old man who developed a large putaminal haemorrhage with subsequent brain herniation and secondary brain stem haemorrhage. When the first examination was performed, he was deeply comatose and all the brain stem reflexes were lost except the cough reflex, whereas spontaneous respiration was preserved. N18 was normally preserved whereas ABRs were lost except bilateral waves I and a questionable left wave II (upper part of fig 3). One hour later, his spontaneous respiration ceased and blood pressure suddenly fell without any circulatory cause, suggesting establishment of brain death. The cough reflex was also lost. Six hours later, the second examination was done, when N18 was completely lost and the ABR showed only a questionable right wave I (lower part of fig 3).

\section{Discussion}

In previous attempts to diagnose brain death by SEPs, the P13/14 potential, which has been mainly attributed to the medial lemniscus, has been used to evaluate brain stem function. However, a major problem was that P13/14 was often preserved even in brain death. ${ }^{3-10}$ Apparent P13/14-like potentials of low amplitude were seen in nine of 12 brain death recordings also in the present series. Previous papers reporting that the "P13" components were preserved in cervicomedullary lesions must have the same relevance. ${ }^{22} 23$ Previous investigators attributed the origin of this remnant P13 to the caudalmost brain stem, foramen magnum, or high cervical cord, ${ }^{1022} 23$ whereas we presented an opinion that it may be related to P11 and dorsal column. ${ }^{21}$ In any case, such a controversy regarding the origin and frequent preservation in brain death (frequent false positives) must have hindered a wide clinical application of P13/14 to the diagnosis of brain death ${ }^{24}$. Wagner et $a l^{9}{ }^{10}$ showed that nasopharyngeal recordings of P14 could 
correctly differentiate between non-brain dead coma and brain death, but the technical difficulty may prevent its routine application. Loss or great reduction of the P13/14 amplitude can be employed as a reliable sign of the establishment of brain death when the course progressing from coma to brain death was followed, as suggested by the present investigation and previous studies. ${ }^{8-10}$ The problem is that it is not always possible to follow such a course, but more often the patient is considered to be brain dead when the first examination is performed (seven of 12 brain death recordings for the present series). If apparent P13/14 is preserved in such cases (six of seven for the present series), even in low amplitude, it is impossible to distinguish such potentials from "true" P13/14 indicating preserved brain stem function.

The N18 potential was first described by Desmedt and Cheron ${ }^{11}$ as a long lasting negativity widely distributed over the scalp, and its generator has long been attributed to brain stem nuclei receiving collaterals from the medial lemniscus, especially the tectal nuclei at the midbrain. ${ }^{25}{ }^{26}$ However, we have shown that N18 was preserved in normal size and shape for patients with medial lemniscal lesions at pontine and medullary levels ${ }^{12-15}$ whereas it was lost in patients with C1-2 dorsal column lesions. ${ }^{1421}$ Especially, in a patient with unilateral medial medullary syndrome in whom subsequent cortical components were severely disturbed unilaterally, N18 after stimulation of the affected side had a similar size and shape to that after stimulation of the unaffected side. ${ }^{15}$ Based on these results, we concluded that at least the major part of $\mathrm{N} 18$ lasting around $20 \mathrm{~ms}$ is principally generated around the medulla oblongata. ${ }^{12-15}$ Medullary origin of N18 has also been supported by other investigators based on findings in patients with brain stem lesions ${ }^{27}{ }^{28}$ or nasopharyngeal recordings. ${ }^{29}$ Intraoperative recordings in humans and direct recordings in animals showed a long lasting potential dipole around the cuneate nucleus similar to N18 in polarity and time course..$^{30-32}$ Andersen $e t a l^{\beta 3}$ showed that in cats this second phase of the cuneate potential with ventrorostral negative polarity is generated by the primary afferent depolarisation of presynaptic terminals of the dorsal column fibres, by contrast with the first phase due to postsynaptic depolarisation of relay cells. Taking these studies into consideration, we presented an opinion that the long lasting negativity of N18 is generated at the cuneate nucleus by the mechanism of primary afferent depolarisation. ${ }^{12-15}$ Recent investigations showed special physiological characteristics of N18, such as conspicuous occlusion induced by adjacent nerve stimulation and lack of inhibition induced by vibration interference, which are compatible with primary afferent depolarisation. ${ }^{34} 35$

The cuneate nucleus is located at the caudal part of the medulla oblongata and is close to the respiratory centre at the medulla oblongata (nucleus solitarius and area reticularis superficialis ventrolateralis ${ }^{36}$ ). Therefore, $\mathrm{N} 18$ is expected to be an indicator of function of the medulla oblongata, especially for the state of vital centres close by.

The present results support this idea. N18 was preserved for all but one non-brain dead recording, whereas it was never found in patients judged to be brain dead. In this sense, N18 was a fully specific and sensitive indicator for the remaining brain stem function (no false positives and rare false negatives). In the patients in whom we followed the course of progression from coma to brain death, loss of N18 seemed to parallel loss of spontaneous respiration as expected from the proximity between the $\mathrm{N} 18$ generator and the respiratory centre, although more intensive observation of the course of sequential loss of individual variables is necessary to confirm the above hypothesis.

The ABRs were indistinguishable from those in brain death (no more than wave I and small wave II) in four of 13 comatose recordings in the present series, indicating a higher percentage of false negatives for detecting remaining brain stem function. This must be due to the fact that ABRs reflecting pontine and midbrain functions cannot reflect medullary function because in three of four recordings in which ABRs failed to show brain stem activities, N18 was preserved together with spontaneous respiration, cough reflex, or decerebrate response. The last result further supports our hypothesis that N18 is generated at the medulla oblongata.

There was no recording where N18 was preserved when clinical signs indicated brain death. Namely, it never happened that N18 could detect remaining brain stem function in the patient clinically diagnosed as brain dead, at least within the present series. In this sense, the clinical determination of brain death was sufficiently safe and N18 gave no additional information. However, this does not mean that N18 has no value. The situation was the same for ABRs because there was no recording in the present series where ABRs showed preserved brain stem functions (preservation of III or later waves) despite the clinical diagnosis of brain death.

As a principle, the diagnosis of brain death is a rule out diagnosis and each clinical or neurophysiological variable can evaluate the function of only a part of the brain stem or the whole brain. Namely, it is an inherent limitation of not only neurophysiological ${ }^{24}$ but also clinical indices used for the diagnosis of brain death that no single index is so powerful that it can unequivocally diagnose brain death by itself. Accordingly, we have no reason to exclude an index which can reliably and non-invasively evaluate an aspect of brain stem function. There is actually a report of rare cases in which evoked potentials excluded brain death despite clinical diagnosis of the event. ${ }^{37}$ An index which gives rare false negatives and no false positives, such as N18, must be especially useful because its preservation definitely excludes brain death and its loss strongly suggests brain death. In any event, it is of value that we have obtained an electrophysiological measure which is 
considered to be an indicator of medullary function. Further investigation is necessary to establish the usefulness of N18 as a new criterion of brain death.

This study is supported by Grants-in-Aid for Scientific Research (09670669) from the Ministry of Education, Science, Sports and Culture of Japan and by The Nakabayashi Trust for ALS Research.

1 Star A. Auditory brain-stem responses in brain death. Brain 1976;99:543-54.

2 Goldie WD, Chiappa KH, Young RR, et al. Brainstem auditory and short-latency somatosensory evoked responses in brain death. Neurology 1981;31:248-56.

3 Facco E, Casartelli Liviero M, Munari M, et al. Short latency evoked potentials: new criteria for brain death? $\mathcal{f}$ Neurol Neurosurg Psychiatry 1990;53:351-3.

4 Machado C, Valdes P, Garcia-Tigera J, et al. Brain-stem auditory evoked potentials and brain death. Electroencephalogr Clin Neurophysiol 1991;80:392-8.

5 Shiogai T. Evaluation of electrophysiological neuromonitoring in the diagnosis of brain death: part 1. brainstem auditory evoked potentials. Brain and Nerve (Tokyo) 1989;41: 73-83.

6 Anziska BJ, Cracco RQ. Short latency somatosensory evoked potentials in brain dead patients. Arch Neurol 1980 evoked poten

7 Belsh JM, Chokroverty S. Short-latency somatosensory evoked potentials in brain-dead patients. Electroencephalog Clin Neurophysiol 1987;68:75-8.

8 Buchner H, Ferbert A, Hacke W. Serial recording of median nerve stimulated subcortical somatosensory evoked potentials (SEPs) in developing brain death. Electroencephalogr Clin Neurophysiol 1988;69:14-23.

9 Wagner W. SEP testing in deeply comatose and brain dead patients: the role of nasopharyngeal, scalp and earlobe derivations in recording the P14 potential. Electroencephalogr Clin Neurophysiol 1991;80:352-63.

10 Wagner W. Scalp, earlobe and nasopharyngeal recordings of the median nerve somatosensory evoked P14 potential in coma and brain death: detailed latency and amplitude analysis in 181 patients. Brain 1996;119:1507-21.

11 Desmedt JE, Cheron G. Non-cephalic reference recording of early somatosensory potentials to finger stimulation in adult or aging normal man: differentiation of widespread N18 and contralateral N20 from the prerolandic P22 and N30 components. Electroencephalogr Clin Neurophysiol

12 Sonoo M, Shimpo T, Genba K, et al. Origin of upper cervical N13 (ucN13) and widespread N18 in the median nerve SEP. Electroencephalogr Clin Neurophysiol 1990;76:106P.

13 Sonoo M, Sakuta M, Shimpo T, et al. Widespread N18 in median nerve SEP is preserved in a pontine lesion. Electroencephalogr Clin Neurophysiol 1991;80:238-40.

14 Sonoo M, Genba K, Zai W, et al. Origin of the widespread N18 in median nerve SEP. Electroencephalogr Clin Neurophysiol 1992;84:418-25.

15 Sonoo M, Hagiwara H, Motoyoshi Y, et al. Preserved widespread N18 and progressive loss of P13/14 of median nerve SEPs in a patient with unilateral medial medullary syndrome. Electroencephalogr Clin Neurophysiol 1996;100: 488-92.

16 Takeuchi K, Takeshita H, Takakura K, et al. Evolution of criteria for determination of brain death in Japan. Acta Neurochir (Wien) 1987;87:93-8.

17 Belsh JM, Blatt R, Schiffman PL. Apnea testing in brain death. Arch Intern Med 1986;146:2385-8.

18 Ropper AH, Kennedy SK, Russell L. Apnea testing in the diagnosis of brain death: clinical and physiological observations. F Neurosurg 1981;55:942-6.
19 American Electroencephalographic Society. Guideline nine: guidelines on evoked potentials. 7 Clin Neurophysiol 1994:1:40-73.

20 Sonoo M, Shimpo T, Genba K, et al. Posterior cervical N13 in median nerve SEP has two components. Electroencephalogr Clin Neurophysiol 1990;77:28-38.

21 Sonoo M, Genba-Shimizu K, Mannen T, et al. Detailed analysis of the latencies of median nerve somatosensory evoked potential components, 2: analysis of subcomponents of the P13/14 and N20 potentials. Electroencephalogr Clin Neurophysiol 1997;104:296-311.

22 Restuccia D, Di Lazzaro V, Valeriani M, et al. Origin and distribution of P13 and P14 far-field potentials after median nerve stimulation. Scalp, nasopharyngeal and neck recording in healthy subjects and in patients with cervical and cervico-medullary lesions. Electroencephalogr Clin Neurophysiol 1995;96:371-84

23 Valeriani M, Restuccia D, Di Lazzaro V, et al. The scalp to earlobe montage as standard in routine SEP recording: comparison with the non-cephalic reference in patients with lesions of the upper cervical cord. Electroencephalogr Clin Neurophysiol 1998;108:414-21.

24 Chatrian GE, Bergamasco B, Bricolo A, et al. IFCN recommended standards for electrophysiologic monitoring in comatose and other unresponsive states. Report of an IFCN committee. Electroencephalogr Clin Neurophysiol 1996;99:103-22.

25 Mauguiére F, Desmedt JE, Courjon J. Neural generators of $\mathrm{N} 18$ and P14 far-field somatosensory evoked potentials studied in patients with lesion of thalamus or thalamocortical radiations. Electroencephalogr Clin Neurophysiol 1983;56: 283-92.

26 Mauguiére F, Desmedt JE. Bilateral somatosensory evoked potentials in four patients with long-standing surgical hemispherectomy. Ann Neurol 1989;26:724-31.

27 Raroque Jr HG, Batjer H, White C, et al. Lower brain-stem origin of the median nerve N18 potential. Electroencephalogr Clin Neurophysiol 1994;90:170-2.

28 Noël P, Ozaki I, Desmedt JE. Origin of N18 and P14 far-fields of median nerve somatosensory evoked potentials studied in patients with a brain-stem lesion. Electroencephalogr Clin Neurophysiol 1996;98:167-70.

29 Tomberg C, Desmedt JE, Ozaki I, et al. Nasopharyngeal recording of somatosensory evoked potentials document the medullary origin of the N18 far-field. Electroencephalogr Clin Neurophysiol 1991;80:496-503.

30 Andersen P, Eccles JC, Schmidt RF, et al. Slow potential waves produced in the cuneate nucleus by cutaneous volleys and cortical stimulation. F Neurophysiol 1964;27:7891

31 Suzuki I, Mayanagi Y. Intracranial recording of short latency somatosensory evoked potentials in man: identification of origin of each component. Electroencephalogr Clin Neurophysiol 1984;59:286-96.

32 Morioka T, Tobimatsu S, Fujii K, et al. Origin and distribution of brain-stem somatosensory evoked potentials in humans. Electroencephalogr Clin Neurophysiol 1991;80:2217 .

33 Andersen P, Eccles JC, Schmidt RF, et al. Depolarization of presynaptic fibers in the cuneate nucleus. $\mathcal{F}$ Neurophysiol 1964;27:92-106.

34 Manzano GM, Negrão N, Nóbrega JAM. The N18 component of the median nerve SEP is not reduced by vibration. Electroencephalogr Clin Neurophysiol 1998;108:259-66.

35 Manzano GM, Negrão N, Nóbrega JAM. Some functional observations related to the N18 component of the median nerve SEP. Electromyogr Clin Neurophysiol 1998;38:440-5.

36 Long SE, Duffin J. The medullary respiratory neurons: a review. Can 7 Physiol Pharmacol 1984;62:161-82.

37 Facco E, Baratto F, Munari M, et al. Short latency evoked potentials in the diagnosis of brain death. Electroencephalogr Clin Neurophysiol 1997;103:39. 